\title{
Indicadores bibliométricos de cooperação científica internacional em bioprospecção
}

Ricardo Arcanjo de Lima;

\begin{abstract}
Bacharel em Biblioteconomia e Ciência da Informação pela Universidade Federal de São Carlos (2003); Mestrando em Política Científica e Tecnológica pela Universidade Estadual de Campinas.
\end{abstract}

Lea Maria Leme Strini Velho;

Graduada em Engenharia Agronômica pela Universidade Estadual Paulista Júlio de Mesquita Filho (1976); Mestre em Agronomia (Produção Vegetal) pela Universidade Estadual Paulista Júlio de Mesquita Filho (1979); Doutorado em Política Científica e Tecnológica - SPRU, University of Sussex, RU (1985); Professora titular da Universidade Estadual de Campinas.

Leandro Innocentini Lopes de Faria;

Graduado em Engenharia de Materiais pela Universidade Federal São Carlos (1994); Mestre em Ciência e Engenharia dos Materiais pelo Universidade Federal São Carlos (1997); Doutor em Sciences de L'information Et Communication pela Universite d'Aix-Marseille III (Droit, Econ. et Sciences) (2001); Professor adjunto da Universidade Federal de São Carlos.

Busca observar os fatores que influem nas relações de colaboração científica de uma atividade de pesquisa multidisciplinar - a área de bioprospecção - como afinidades econômicas, culturais e geográficas. Apresenta e analisa indicadores bibliométricos de co-autoria que abrangem o período de 1986 a 2006, computados a partir de uma base bibliográfica internacional e multidisciplinar, o Science Citation Index.

Palavras-chave: Cooperação científica; Bibliometria; Bioprospecção. 


\title{
Bibliometric Indicators of International Scientific Cooperation in Bioprospection
}

\begin{abstract}
The current article aims at observing the factors that influence in the relations of scientific collaboration of a multidisciplinary research activity - the area of bioprospection - such as economical, cultural and geographical similarities. Bibliometric indicators of $\mathrm{CO}^{-}$ authorship are presented and analyzed, gathered from an international and multidisciplinary bibliographic basis, the Science $\quad$ Citation Index, from 1986 to 2006.
\end{abstract}

Key-words: Scientific cooperation; Bibliometry; Bioprospection.

Recebido em 04.01.2007 Aceito em 14.03.2007

\section{Introdução}

A ciência tem uma série de características que lhe imprimem um caráter internacional: o conhecimento gerado é codificado em periódicos que estão disponíveis em todo mundo, os pesquisadores se encontram em reuniões internacionais, viajam para trabalhar em laboratórios de outros países e freqüentemente envolvem colegas estrangeiros em seus projetos de pesquisa. Essas atividades têm se intensificado significativamente nos últimos anos, resultando em notável aumento da colaboração científica internacional. No início do século $X X$, a colaboração científica envolvia pesquisadores trabalhando na mesma instituição e, em alguns casos, colegas de diferentes instituições na mesma região ou país, como observa Wagner (2004). Na contemporaneidade, a colaboração internacional cresceu muito mais rapidamente do que a colaboração entre instituições dentro das fronteiras nacionais - no período entre 1988 a 2001, exemplificando, enquanto a proporção de artigos co-autorados em geral na base de dados do Science Citation Index aumentou 8\%, o número de artigos em co-autoria internacional mais do que duplicou (NATIONAL SCIENCE BOARD, 2006). Por essa razão, aliada ao fato de que os artigos envolvendo autores de diferentes países tendem a ter maior impacto, os estudiosos da questão têm afirmado que a cooperação internacional é um requisito da ciência moderna de qualidade (MERLIN e PERSSON, 1996), e um dos principais fatores que determinam o sucesso na pesquisa científica (LECLERC et al, 1992).

Para Luukkonen, Persson e Silvertsen (1992) são três os fatores que têm influenciado a cooperação científica internacional: a) os econômicos, ligados ao custo do projeto de pesquisa, levam os pesquisadores a buscar a colaboração com colegas de outros países, de forma a viabilizar 
financeiramente os projetos pela partilha dos custos. Tais fatores abrangem as pesquisas da chamada big science - atividades científicas que requerem alto investimento financeiro (aquisição de instrumentos caros e construção de laboratórios complexos como os aceleradores de partículas) e ocorrem mais freqüentemente entre as instituições dos países mais ricos; b) os cognitivos, relacionados ao acesso ao conhecimento, dizem respeito à busca dos pesquisadores por cooperação para complementar seu conhecimento em determinada área e assim agregar conhecimentos essenciais para a execução de seus projetos. Como exemplo podemos tomar as redes multidisciplinares de pesquisa nas áreas de genética, biotecnologia e nanotecnologia; c) os sociais, que são os fatores ligados à rede de relacionamento dos pesquisadores, levando-os a buscar cooperação com colegas com os quais mantenham relações tanto profissionais como pessoais, ou por afinidade temática, emocional ou ideológica. Um bom exemplo é a relação que se estabelece entre orientador e orientado, parceria essa que tende a se manter mesmo após o vínculo formal desaparecer.

Além dos fatores apresentados acima, Georghiou (1998), apresenta os benefícios que agem como motivação para a cooperação entre pesquisadores. Tais benefícios podem ser diretos e indiretos:

Diretos:

- Acesso a conhecimento, especialistas e habilidades em ciência e tecnologia;

- Acesso a lugares únicos, grupos populacionais específicos;

- Divisão de custos e riscos;

- Solução para questões globais e estabelecimento de padrões internacionais.

Indiretos:

- Motivações estratégicas, por exemplo, situações em que a colaboração é motivada por fatores externos de natureza política, econômica ou cultural.

Portanto, diversos aspectos influenciam o aumento da cooperação científica, mas a incidência dos fatores mencionados tem atingido áreas de conhecimento e países avançados e em desenvolvimento de formas diferentes. Em termos de áreas do conhecimento, a colaboração internacional, vista em termos globais, é muito mais comum nas ciências exatas e da terra (em torno de $35 \%$ das publicações) do que nas ciências humanas e sociais, onde os artigos em co-autoria internacional representaram apenas $14 \%$ do total das publicações de 2003 . Vale notar também que, no período entre 1988 e 2003, o maior crescimento em termos de cooperação internacional se deu nas engenharias/tecnologia, refletindo a tendência atual de parcerias entre empresas e entre setor público de pesquisa e setor produtivo no nível global. Esse padrão, entretanto, reflete a realidade dos países industrializados, já que esses, considerados em conjunto, publicam mais de $80 \%$ dos artigos indexados no Science Citation Index - SCI - (NATIONAL SCIENCE BOARD, 2006). Quando se analisa, separadamente, a produção dos países em 
desenvolvimento, a presença da cooperação internacional é mais marcante nas ciências da vida, particularmente na biomedicina e nas ciências agrárias, indicando a prioridade colocada nessas áreas pelos governos destes países, assim como por financiadores internacionais, principalmente as agências de cooperação para o desenvolvimento (WAGNER et al, 2001).

A motivação dos pesquisadores para se envolver em atividade de colaboração internacional também varia - os provenientes dos países avançados tendem a ser movidos por fatores tais como o acesso ao conhecimento e tecnologias inéditas em seus países de origem, interesses comuns e partilha de financiamento. Já a colaboração dos países avançados com os países em desenvolvimento tende a acontecer principalmente pela necessidade dos primeiros em ter acesso a lugares, grupos populacionais e conhecimentos tradicionais disponíveis apenas nos últimos.

No caso específico da área de bioprospecção, que consiste em termos amplos, na identificação e avaliação de material biológico encontrado na natureza para a obtenção de novos produtos ou processos (ARTUSO, 2002), a colaboração científica internacional é motivada pelo interesse dos países avançados em ter acesso aos recursos da biodiversidade e conhecimentos tradicionais das populações dos países em desenvolvimento (VELHO, 2002).

Sendo assim, este artigo busca compreender a cooperação internacional nas atividades científicas ligadas à bioprospecção, através da análise bibliométrica de dados de co-autoria em papers. Parte-se da premissa que essa análise possa identificar os países que estabelecem parcerias nesta área, assim como a intensidade em que essa cooperação tem variado no tempo. A área de bioprospecção foi escolhida devido à sua crescente importância em nível mundial, sobretudo após a ratificação da Convenção sobre a Diversidade Biológica - CDB.

O texto está estruturado em três seções principais. Primeiramente, faz-se uma breve caracterização sobre o uso da análise bibliométrica no estudo da cooperação científica. Em seguida, apresenta-se a metodologia de coleta dos dados, extraídos do Science Citation Index no período de 1986-2006. Por fim, os resultados obtidos são dispostos em gráficos e mapas bibliométricos de co-autoria e analisados à luz da literatura relevante e do contexto.

\section{Indicadores bibliométricos de cooperação científica}

Em anos recentes, como afirma Katz (1994), a comunidade científica e os analistas de política científica têm se interessado em entender o crescente fenômeno da colaboração em pesquisa. Vários autores analisam essa cooperação, buscando identificar os fatores (LUUKKONEN; PERSSON; SILVERTSEN, 1992), e as motivações (GEORGHIOU, 1998) responsáveis por tal crescimento. Assim, a cooperação científica internacional tem sido objeto de estudo de disciplinas como a sociologia, a política e a bibliometria. (WAGNER, 2004). 
Esta última é a perspectiva adotada por esse artigo. Quando se fala de cooperação científica na visão da bibliometria, devemos ter em mente que quase sempre estamos nos referindo à análise de trabalhos publicados em co-autoria.

Para Leclerc et al (1992), os artigos em co-autoria transnacional são um importante indicador de cooperação científica internacional, lançando uma luz nos canais da ciência mainstream e provendo informações concernentes às tendências e ao grau de atividade científica. Estudos semelhantes foram desenvolvidos por Price e Beaver (1966), que usaram as relações de co-autoria para investigar as estruturas sociais e sua influência na ciência e mais especificamente na comunicação das redes. Stokes e Hartley (1989) examinaram, também através de dados de coautoria de artigos, caminhos para identificar os cientistas mais influentes, as chamadas figuras chave.

Apesar da análise bibliométrica de co-autoria ser bastante usada na construção de indicadores de cooperação científica, a validade desta técnica para estudar a cooperação científica internacional ainda não é consensual. A co-autoria retrata apenas uma parte - aquela mais formal das várias atividades envolvidas nos trabalhos colaborativos, assim como das contribuições de cada autor na atividade de pesquisa em questão. Uma outra fonte importante de registro da cooperação, e também registrada na literatura científica, são os acknowledgements ou agradecimentos, como nos informa Roa Cellis (2002). Por isso ao analisarmos dados de co-autoria na intenção de construir indicadores de cooperação, devemos ter em mente algumas opções a considerar dependendo do tipo de questão a ser levantada (MERLIN e PERSSON, 1996):

- Nível de agregação ou foco do estudo;

- Análise de artigos, estudo da co-autoria nacional ou internacional;

- Análise das redes, a co-autoria pode ser usada na construção de redes de pesquisa e comunicação científica.

A definição prévia do foco e o recorte geográfico do estudo são importantes para a escolha apropriada do instrumental bibliométrico. Nesse sentido, vale apontar que o campo autor é alvo de críticas, uma vez que nem toda colaboração resulta em artigo e, por outro lado, co-autoria nem sempre indica colaboração, existindo todo um processo de negociação entre os colaboradores para decisão sobre quem vai assinar determinado artigo. Além do mais, esse processo de negociação varia entre áreas do conhecimento e reflete processos sociais condicionados pelo contexto histórico e social em que se localizam os colaboradores.

Apesar de não abranger todos os aspectos e formas possíveis da cooperação científica, excluindo, por exemplo, o conhecimento tácito transmitido entre pesquisadores, e apesar das limitações apontadas acima, a análise bibliométrica de co-autoria é reconhecidamente o método mais difundido de estudo quantitativo da cooperação científica, sendo 
usado pela maior parte dos autores que trabalham com esse tema (LUUKKONEN et al, 1993) ${ }^{1}$; MELIN, 1999) ${ }^{2}$ e (GLANZEL, 2001) ${ }^{3}$. Por essa razão, e pelo fato de que a análise de co-autoria permite analisar um importante meio de comunicação científica, qual seja, os artigos publicados em periódicos especializados, optamos por esse caminho nesse artigo.

\section{Metodologia}

A fonte dos dados usada no estudo é à base de dados multidisciplinar Science Citation Index - SCI -. A escolha deve-se à sua abrangência e representatividade, sendo o SCI responsável pela indexação de documentos das mais diversas áreas do conhecimento, entre elas as de agricultura, tecnologia dos alimentos, bioquímica, biologia, ciências médicas, química, computação, ciências ambientais, genética, microbiologia, farmacologia, veterinária e zoologia (THOMSON, 2004), áreas afins ao foco desse estudo - a bioprospecção.

Outro fator crucial para a escolha do Science Citation Index é o método de indexação da base de dados, que registra os dados bibliográficos completos para cada documento incorporado, incluindo os resumos originais em inglês, os endereços dos autores e editores e as referências bibliográficas citadas em cada artigo (TESTA, 1998). O processo de indexação dos dados de afiliação de todos os autores é fundamental para o estudo da cooperação científica internacional através da co-autoria.

Os descritores para recuperação dos papers relativos à bioprospecção foram escolhidos a partir do teste de diversas estratégias de busca, associando verbos que indicam atividade prospectiva como extract e prospecting a elementos da biodiversidade.

Essas estratégias de caráter exploratório tinham o objetivo de refinar os descritores, devido à complexidade de se trabalhar com áreas multidisciplinares. Uma vez definidos os descritores, os mesmos foram usados em estratégias combinadas na modalidade de busca Advanced Search do Science Citation Index.

A busca, restrita aos anos de 1986 a 2006, recuperou 20.439 artigos que sofreram tratamento bibliométrico automatizado através do software VantagePoint, software de Data Mining que permite a extração de conhecimento de bases de dados.

A escolha do período analisado teve o objetivo de limitar o estudo em duas décadas, sem perda de atualidade dos dados.

\section{Resultados e discussão}

1 LUUKKONEN, T.; TIJSEEN, R. J. W.; PERSSON, O.; SIVERTSEN, G. The measurement of international scientific collaboration. Scientometrics, v. 28, 1993.

2 MELIN, G. Impact of national size on research collaboration. Scientometrics, v. 46, 1999.

3 GLÄNZEL, W. National characteristics in international scientific co-authorship relations, Scientometrics, v. 51, 2001. 
A partir dos dados coletados no Science Citation Index foram produzidos gráficos e mapas de co-autoria que vão nos ajudar a compreender a atividade científica na área de bioprospecção.

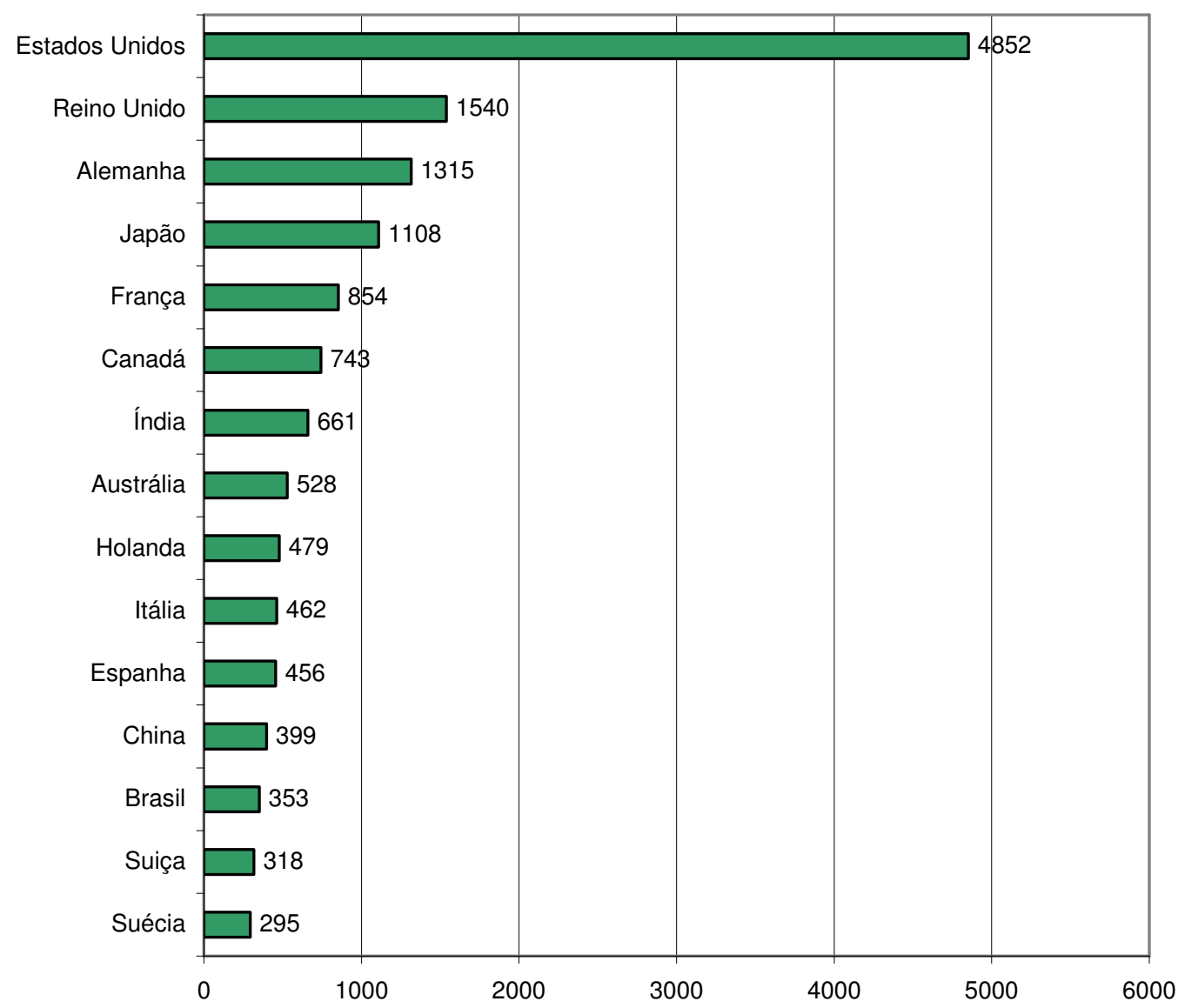

GRÁFICO 1 - Países que mais publicaram na área de bioprospecção no período de 1986-2006, segundo dados extraídos do SCI

Fonte: SCI, via Web of Science (2006)

No GRÁF. 1 observa-se o ranking dos países que mais publicaram na área de bioprospecção no período estudado. O GRÁF. 1 indica uma clara predominância dos países avançados do Norte, em especial dos Estados Unidos, que lideram o ranking com 4,852 artigos indexados e dos países da Europa Ocidental.

Outras nações bem colocadas são Japão e Canadá que, através de institutos de pesquisa (Hitachi Foundation e International Development Research Centre), fazem parte,- como a maioria dos países representados no gráfico, do ISAAA - International Service for the Acquisition of Agribiotech Applications. O objetivo do ISAAA é facilitar a transferência e adoção de aplicações biotecnológicas para os países em desenvolvimento, além de construir parcerias entre instituições do Sul com o setor privado do Norte, e de fortalecer a cooperação Sul-Sul. Essa iniciativa tem financiado cerca de uma dúzia de projetos, contratados e implementados ou ainda em desenvolvimento (VELHO e VELHO, 2001). 

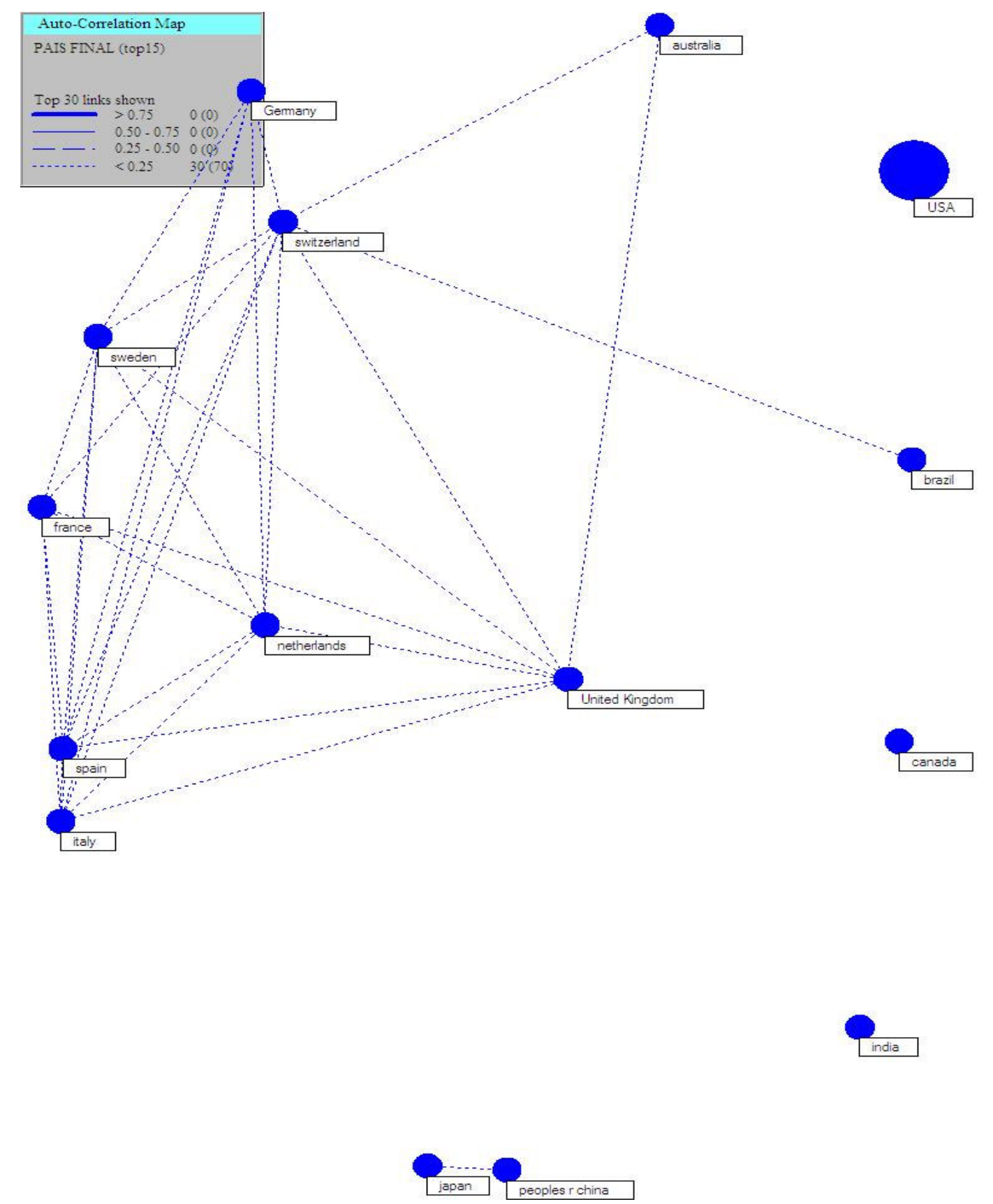

FIGURA 1 - Mapa de Co-autoria em Bioprospecção no período de 1986-2006, segundo dados extraídos do SCI.

Fonte: SCI, via Web of Science (2006)

Outro fato que chama a atenção no GRÁF. 1 é a presença do Brasil, China e Índia, membros dos chamados países megadiversos - grupo composto por 14 países que possuem juntos, cerca de $70 \%$ da diversidade biológica do mundo (NANNI, 2003). Tal fato nos remete ao interesse crescente dos países ricos em biodiversidade em pesquisar e explorar seus próprios recursos genéticos, em especial aquelas nações consideradas economicamente emergentes.

Na FIG. 1, como pode ser observado, foram mapeadas as ligações existentes entre os países que mais publicaram em uma faixa de co- 
relação 0,30, considerada representativa segundo o coeficiente de Pearson ${ }^{4}$. Podemos observar que praticamente as ligações se restringiram aos países da Europa Ocidental, com exceções da colaboração entre Reino Unido e Austrália, China e Japão, e Brasil e Suíça. Os vínculos de coautoria refletem as afinidades econômicas, culturais e geográficas desses países, em especial no caso europeu onde todos os países, com exceção da Suíça, são membros da União Européia. Tal fato também é evidente na cooperação entre Reino Unido e Austrália que mantêm laços históricos e políticos.

Outro aspecto interessante é a ligação entre Brasil e Suíça, sendo o primeiro um país megadiverso, que estabelece parceria com a segunda, sede de importantes indústrias do setor farmacêutico como a Novartis, Pharmacia Aktiebolag e F. Hoffman-la Roche. Tais vínculos, detectados por parcerias em artigos científicos, podem indicar uma colaboração estratégica entre pesquisadores dos dois países, qual seja, a realização de pesquisas bioprospectivas de utilidade para a indústria farmacêutica.

Os Estados Unidos, país que lidera o ranking em número de publicações em bioprospecção, não mantiveram cooperação internacional proporcional com os demais países analisados. Este fenômeno pode indicar que o país mantém vínculos nacionais mais fortes em detrimento dos vínculos internacionais na sua produção científica em bioprospecção. Vale ressaltar, no entanto, que esse dado não exclui os Estados Unidos de ser um dos principais parceiros de cooperação de países com volume menor de publicação.

A América Latina abriga oito das 14 nações consideradas megadiversas, razão pela qual no QUAD. 2, recortamos em uma matriz as co-autorias envolvendo os países latino-americanos e seus parceiros internacionais. A matriz se limitou a destacar apenas os países que apresentaram 20 papers ou mais, indexados na base de dados no período estudado.

Observa-se na matriz que o principal parceiro dos países latinoamericanos são os Estados Unidos e os países membros da União Européia, em detrimento de países mais próximos em termos de realidade socioeconômica localizados em outras regiões do globo como Ásia, África, Oceania e restante do continente americano, abarcados sob o rótulo outros países.

Tal fato já era esperado, uma vez que as nações latino-americanas biodiversas tendem a buscar parceiros do norte, detentores de conhecimento técnico-científico e de recursos financeiros, para auxiliá-las na exploração de sua biodiversidade - em especial de países da Europa e dos Estados Unidos, com quem a região mantém antigos laços históricos, culturais e geopolíticos.

4 O coeficiente de correlação de Pearson também chamado de coeficiente de correlação produtomomento ou simplesmente de $r$ de Pearson mede o grau da correlação (e a direcção dessa correlação - se positiva ou negativa) entre duas variáveis de escala métrica (intervalar ou de rácio). Usado justamente para não nos enganarmos com os números absolutos e analisarmos as ligações proporcionais. 
Com a exceção de Brasil, Argentina e Venezuela, que apresentam mais papers em colaboração com países membros da União Européia, os Estados Unidos é o principal parceiro dos países latino-americanos. O peso dessa parceria é visível nos casos do Peru, Chile e Panamá. O caso peruano merece destaque, uma vez que, dos 20 papers produzidos por autores daquele país, 11 apresentaram ligações de co-autoria com os Estados Unidos. Essa forte ligação pode ser um reflexo da participação do Peru nos projetos ICBG - Grupos Internacionais de Cooperação em Biotecnologia -, onde importantes institutos de pesquisa do Peru, como o museu de História Natural e a Universidade Cayetano cooperam com universidades e empresas norte-americanas, principalmente na área de fontes botânicas para novos medicamentos (VELHO e VELHO, 2001).

Apesar da liderança americana, com 133 ligações de co-autoria com países latino-americanos, a matriz nos mostra a existência de um equilíbrio com o pólo europeu, uma vez que as ligações européias com autores latino-americanos somam 119. O Brasil é um bom exemplo desse caso - a participação européia e americana com o país difere por apenas dois papers.

QUADRO 2 - Matriz de co-autoria internacional - Países Latinoamericanos, no período de 1986-2006, segundo dados extraídos do SCI.

\begin{tabular}{|c|c|c|c|c|}
\hline \multirow{2}{*}{ Total de Artigos } & \multirow{2}{*}{ Países } & \multicolumn{3}{|c|}{ Parceiros de Co-autoria } \\
\cline { 3 - 5 } & & União Européia & Estados Unidos & Outros Países \\
\hline 353 & Brasil & 36 & 34 & 9 \\
\hline 142 & México & 23 & 31 & 13 \\
\hline 111 & Argentina & 25 & 8 & 7 \\
\hline 44 & Chile & 8 & 17 & 1 \\
\hline 33 & Colombia & 5 & 9 & 7 \\
\hline 28 & Panamá & 7 & 11 & 3 \\
\hline 25 & Costa Rica & 4 & 9 & 3 \\
\hline 20 & Peru & 5 & 11 & 3 \\
\hline 20 & Venezuela & 6 & 3 & \\
\hline
\end{tabular}

Fonte: SCI, via web of science (2006)

No QUAD. 3 a matriz aborda as co-autorias existentes entre países latino-americanos, tomando também como critério o mínimo de 20 papers indexados, tendo como objetivo analisar a colaboração regional na área de bioprospecção. A cooperação em número de papers entre os latinoamericanos é relativamente pequena - no caso brasileiro, de todos 353 artigos publicados, apenas 8 foram em co-autoria com países latinoamericanos. O mesmo pode ser dito de outros países como México (8) e 
Argentina (10) - mas, em termos proporcionais, países como Argentina, Chile, Colômbia e outros cooperam significativamente com os países da região.

QUADRO 3 - Matriz de co-autoria regional - Países Latino-americanos, no período de 1986-2006, segundo dados extraídos do SCI.

\begin{tabular}{|c|c|c|c|c|c|c|c|c|c|}
\hline \multirow{2}{*}{ Países } & \multicolumn{7}{|c|}{ Parceiros de Co-autoria } \\
\cline { 2 - 12 } & Brasil & México & Argentina & Chile & Colombia & Panamá & $\begin{array}{c}\text { Costa } \\
\text { Rica }\end{array}$ & Peru & Venezuela \\
\hline Brasil & $\mathbf{3 5 3}$ & 1 & 2 & 1 & & & 2 & 2 & \\
\hline México & 1 & $\mathbf{1 4 2}$ & 2 & 2 & 1 & & & 1 & 1 \\
\hline Argentina & 2 & 1 & $\mathbf{1 1 1}$ & 3 & 1 & 2 & 1 & & \\
\hline Chile & 1 & 2 & 3 & $\mathbf{4 4}$ & & & & & \\
\hline Colombia & & 1 & 1 & & $\mathbf{3 3}$ & 1 & 2 & 2 & \\
\hline Panamá & & & 2 & & 1 & $\mathbf{2 8}$ & 1 & & \\
\hline $\begin{array}{c}\text { Costa } \\
\text { Rica }\end{array}$ & 2 & & 1 & & 2 & 1 & $\mathbf{2 5}$ & & \\
\hline Peru & 2 & 1 & & & 2 & & & $\mathbf{2 0}$ & \\
\hline Venezuela & & 1 & & & & & & & $\mathbf{2 0}$ \\
\hline
\end{tabular}

Fonte: SCI, via Web of Science (2006)

É interessante ressaltar que a área destacada na matriz indica o total de artigos do país indexados e não em co-autoria, podendo-se tomar como exemplo o Peru - dos 20 artigos indexados no período analisado, 5 foram em colaboração com outros países latino-americanos.

No caso brasileiro, se observamos o QUAD. 2, veremos que dos 353 artigos indexados do país, 79 deles são em co-autoria com outros países, tornando os 10 artigos em colaboração com seus vizinhos mais representativos.

Através dos gráficos, mapas e matrizes podemos observar que o Brasil se destaca no contexto latino-americano em volume de publicações indexadas, sendo o único país latino-americano a figurar no GRAF. 1, fazendo-se conveniente explicitar na FIG. 2 as ligações de co-autoria na área de bioprospecção entre as instituições de pesquisa do país que mais publicaram no período estudado.

$\mathrm{Na}$ FIG.2 identifica-se a existência de um cluster envolvendo as universidades estaduais dos estados de São Paulo e Paraná Universidade de São Paulo, Universidade Estadual de Campinas, Universidade Estadual Paulista, Universidade Estadual de Maringá e Universidade Estadual de Londrina, instituições estas envolvidas direta ou indiretamente em projetos ligados à Mata Atlântica do programa Biota 
financiado pela $\mathrm{FAPESP}^{5}$, que desenvolve pesquisas de caracterização, conservação e uso sustentável da biodiversidade.

FIGURA 2 - Mapa de Co-autoria - Instituições brasileiras, no período de 1986-2006, segundo dados extraídos do SCI.

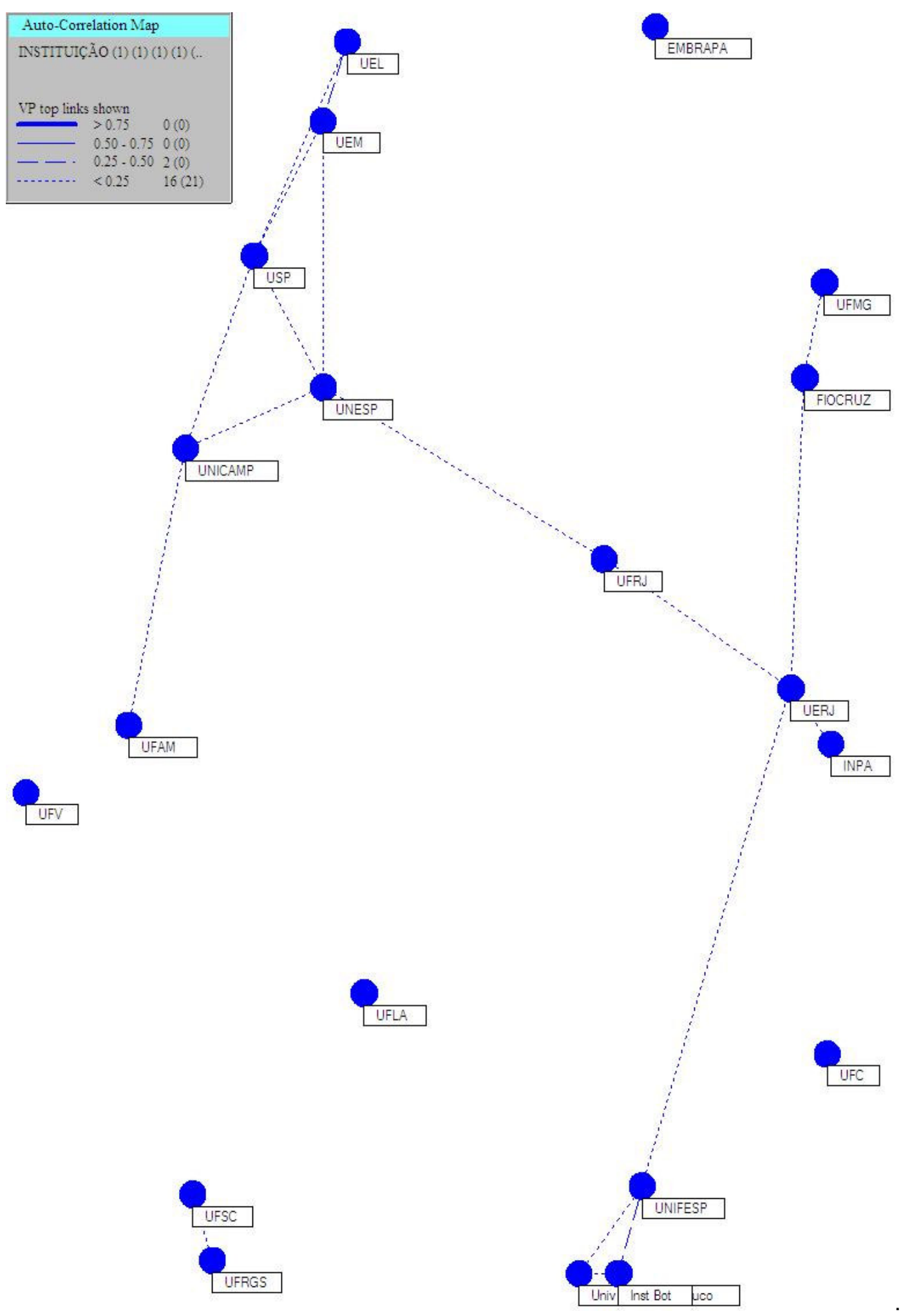


Outro agrupamento (aparentemente sem vínculo formal entre si) envolve o Instituto de Botânica de São Paulo, Universidade Federal de São Paulo e Universidade Federal de Pernambuco, instituições com tradição acadêmica em pesquisas nas áreas de ciências biológicas e da saúde, com destaque para o Instituto Botânico, que mantém programas específicos ligados à biodiversidade e à bioprospecção.

Outras colaborações envolvem Universidade Federal de Minas Gerais - FIOCRUZ, Universidade Estadual do Rio de Janeiro - FIOCRUZ, Universidade Estadual de Campinas - Universidade Federal do Amazonas, Instituto Nacional de Pesquisa da Amazônia - Universidade Estadual do Rio de Janeiro, Universidade Federal de Santa Catarina - Universidade Federal do Rio Grande do Sul, Universidade Estadual Paulista Universidade Federal do Rio de Janeiro, Universidade Estadual do Rio de Janeiro - Universidade Federal do Rio de Janeiro e Universidade Estadual do Rio de Janeiro - Universidade Federal de São Paulo. Constata-se um predomínio dos vínculos entre instituições das regiões Sudeste e Sul do Brasil, regiões essas mais desenvolvidas economicamente e que, provavelmente, destinaram mais recursos para pesquisas na área. Neste panorama, destaca-se a participação da Universidade Estadual do Rio de Janeiro que mantém vínculos com quatro instituições, em especial com o Instituto Nacional de Pesquisas da Amazônia - tal proeminência é decorrente da postura da Universidade em trabalhar o tema através de programas de pós-graduação, como o multidisciplinar curso de Meio Ambiente e do Centro de Estudos Ambientais e Desenvolvimento Sustentável.

Outro dado interessante é o isolamento de um instituto de pesquisa do porte da Empresa Brasileira de Pesquisa Agropecuária - EMBRAPA que possui unidades em praticamente todos os estados brasileiros e, provavelmente, responde por uma considerável fatia da produção em bioprospecção na área agrícola, um nicho que tem despertado o interesse das agências internacionais de fomento. Além da EMBRAPA, as universidades Federais de Viçosa e Lavras - outras duas instituições de ensino e pesquisa com forte tradição na área agropecuária - também se encontram isoladas. Apesar de insuficientes para traçar uma tendência da área, os dados aqui analisados podem indicar um viés institucional na pesquisa brasileira realizada nas áreas de bioprospecção, ciências agrárias e veterinárias. É interessante destacar que a FIG. 2 representa apenas as ligações de co-autoria nacionais.

\section{Considerações finais}

A análise dos indicadores aqui apresentados nos permite observar alguns fatores que parecem influenciar as relações de colaboração científica na área de bioprospecção, como as afinidades econômicas, culturais e geográficas. E, apesar de limitada aos países que mais 
publicaram no período analisado, a FIG.1 explicita a dinâmica da cooperação científica, em que países avançados tendem a cooperar mais entre si em detrimento, inclusive, da parceria com países em desenvolvimento mega-diversos.

Dos resultados expostos a partir de uma perspectiva global podemos considerar como um dos resultados mais desalentadores a baixa taxa de colaboração existente na área de bioprospecção entre os países da América Latina e, com a exceção de alguns países, a posição periférica que a região ocupa no panorama da pesquisa mundial nesta área. Essa região, tão rica em recursos biodiversos, necessita de um esforço regional na formulação de políticas científicas e tecnológicas voltadas à bioprospecção, com o objetivo de fomentar a geração e a transferência de tecnologia.

Por fim, é importante mencionar que dentro do sistema de ciência e tecnologia os indicadores bibliométricos são úteis para avaliar resultados do investimento em pesquisa; porém, é arriscado concluir sobre a dinâmica da área de bioprospecção baseado apenas em dados quantitativos, utilizando-se da mensuração como única fonte confiável para retratar a realidade desta área. No entanto, os dados permitem confirmar a interferência de diversos fatores nos vínculos de colaboração estabelecidos na produção do conhecimento científico na área estudada.

\section{Referências}

ARTUSO, A. Bioprospecting, Benefit Sharing, and Biotechnological Capacity Building. World Development, v. 30, n. 8, 2002.

GEORGHIOU, L. Global cooperation in research. Research Policy, v. 27, 1998.

KATZ, J. S. Geographical Proximity and Scientific Collaboration. Scientometrics, v. 31, n. 1, 1994.

LECLERC, M. et al. Scientific co-operation between Canada and the European Community. Science and public Policy, v. 19, n. 1, 1992.

LUUKKONEN, T.; PERSSON, O.; SIVERTSEN, G. Understanding Patterns of International Scientific Collaboration. Science, Technology, \& Human Values, v. 17, n. 1, 1992.

MERLIN, G; PERSSON, O. Studying research collaboration using coauthorship. Scientometrics, v. 36, n. 3, 1996.

NANNI, S. A quem pertence a biodiversidade? Com ciência, Campinas, abr. 2003. Disponível em http://www.comciencia.br/ reportagens/ genetico/gen02.shtml. Acesso em 10 ago. 2006.

NATIONAL SCIENCE BOARD. Science and Engineering Indicators 2006. Arlington: National Science Foundation, 2006. 
CELIS, Adriana Roa. A dinâmica da comunidade científica na produção do conhecimento um estudo da imunologia no Brasil e na Colômbia. 2002. Tese (Doutorado) - Programa de Pós-Graduação em Política Científica e Tecnológica, UNICAMP, Campinas, 2002.

PRICE, D. J. S.; BEAVER, D. B. Collaboration in an invisible college. American Psychologist, v. 21, 1966.

STOKES, T. D.; HARTLEY, J. A. Coauthorship, social structure and influence within especialties. Social Studies of Science, v. 19, 1989.

TESTA, J. A base de dados ISI e seu processo de seleção de revistas. Ciência da Informação, Brasília, v. 27, n. 2, 1998.

VELHO, L. North-South Colaboration and Systems of Innovation. In: NORTH-SOUTH RESEARCH COOPERATION INTERNATIONAL CONFERENCE, 2001, Amsterdam. Anais... Amsterdam: Koninklijke Nederlandse, 2002.

VELHO, P. E.; VELHO, L. S. Biotecnologia e recursos genéticos: ação e cooperação. Brasília: MCT, 2001. Disponível em http://www.mct.gov.br/ upd blob/443.pdf. Acesso em 05 out. 2006.

WAGNER, C. S. International Collaboration in Science: A New Dynamic for Knowledge Creation. 2004. Tese (Doutorado) - Amsterdam School of Communications Research, University of Amsterdam, Amsterdam, 2004. 